\title{
Resonant soft x-ray scattering from structured polymer nanoparticles
}

\author{
Tohru Araki and Harald Ade ${ }^{\text {a) }}$ \\ Department of Physics, North Carolina State University, North Carolina 27695-8202
}

Jeffrey M. Stubbs and Donald C. Sundberg

Nanostructured Polymers Research Center, University of New Hampshire, Durham, New Hampshire 03824

Gary E. Mitchell

The Dow Chemical Company, 1897 Bldg., Midland, Michigan 48667

Jeffrey B. Kortright

Materials Sciences Division, Lawrence Berkeley National Laboratory, Berkeley, California 94720

\author{
A. L. D. Kilcoyne \\ Advanced Light Source, Lawrence Berkeley National Laboratory, Berkeley, California 94720
}

(Received 18 October 2005; accepted 8 August 2006; published online 22 September 2006)

\begin{abstract}
The application of resonant soft x-ray scattering to chemically heterogeneous soft condensed matter materials is presented. Two structured styrene-acrylic polymer composite latex particles $\sim 230 \mathrm{~nm}$ in diameter were utilized to delineate the potential utility of this technique. Angular scans at photon energies corresponding to strong scattering contrast between specific chemical moieties made it possible to infer the effective radii that correspond to the two polymer phases in the nanoparticles. The results show that resonant soft x-ray scattering should be a powerful complementary tool to neutron and hard x-ray scattering for the characterization of structured soft condensed matter nanomaterials. (C) 2006 American Institute of Physics. [DOI: 10.1063/1.2356306]
\end{abstract}

Structured macromolecular materials play an important role in many nanotechnology and biotechnology developments (see, e.g., Sundberg and Durant ${ }^{1}$ and references therein). Owing to their small size scale and complexity a wide range of powerful tools, e.g., $\mathrm{x}-\mathrm{ray}^{2,3}$ and neutron scattering, ${ }^{4,5}$ electron microscopy and scanning probe microscopy, and complementary methods are necessary to infer and delineate morphology and composition. Synthetic composite latex particles, which are composed of two or more homo- or copolymers, have an extraordinary range of applications and are good examples of nanomaterials with internal structures. ${ }^{1}$ Complex dynamics during synthesis and competing interfacial forces lead to complex structures, including many states of incomplete phase separation. ${ }^{1}$ Competent characterization of these materials is often very challenging and sometimes seemingly impossible. ${ }^{6}$ Since the various components of these composite particles are often chemically similar to each other, complete characterization requires both high spatial resolution and high chemical sensitivity. Transmission electron microcopy (TEM) is a technique with high spatial resolution and has made great contributions to date. Due to potential preparation artifacts, "round robin" characterization has been used to arrive at sound morphological models. ${ }^{6}$ A complementary technique that can provide excellent chemical sensitivity, ${ }^{7,8}$ but is presently limited in spatial resolution to $\sim 40 \mathrm{~nm},{ }^{9}$ is scanning transmission x-ray microscopy (STXM).

Complementary to microscopy, scattering methods can provide spatial information of ensemble averages approaching the $\lambda / 2$ limit $(\lambda=\text { wavelength })^{10}$ in a backscattering geometry. ${ }^{2,11}$ Neutron scattering typically requires deuteration. ${ }^{12,13}$ For hard $\mathrm{x}$ rays, scattering intensity is deter-

\footnotetext{
${ }^{\text {a) }}$ Author to whom correspondence should be addressed; electronic mail: harald_ade@ncsu.edu
}

mined by electron density differences, which are often quite low. Resonant (anomalous) X-ray scattering (RXS) can achieve improved chemical differentiation. ${ }^{14-16}$ RXS with soft $\mathrm{x}$ rays has previously focused on the study of magnetic materials, ${ }^{17,18}$ but also offers important advantages for the differentiation of small phases in soft condensed matter materials or for thin film analysis. ${ }^{19}$ Tunable RXS contrast was demonstrated for spherical, single phase polymeric structures embedded in a polymeric matrix ${ }^{20}$ and for block copolymer samples. ${ }^{21}$ In this letter, the utility of RXS to characterize more complex objects such as structured polymeric nanoparticles will be demonstrated, and the results will be discussed in the context of conventional x-ray scattering, STXM, and TEM capabilities.

Two types of styrene-acrylic polymer latex particles synthesized with a two step polymerization process were characterized. Sample A was composed of a poly(methyl methacrylate) (PMMA) first stage polymerization and a second stage of a random copolymer of $30 / 70 \mathrm{w} / \mathrm{w}$ butyl acrylate (BA) and styrene (S) $[\mathrm{P}(\mathrm{BA}-\mathrm{co}-\mathrm{S})]$. The conditions utilized lead to nonspherical particles. ${ }^{6}$ Sample B was composed of a random copolymer first stage of 40/60 w/w methyl acrylate (MA) and MMA [P(MA-co-MMA)] and a second stage polymerization of polystyrene (PS). Although the synthesis conditions were similar to system $\mathrm{A},{ }^{6}$ a more complicated structure resulted, in which phase separation is incomplete and small domains of PS might be dispersed within the seed particle core.

Nanoparticles were cast from a diluted aqueous suspension onto $100 \mathrm{~nm}$ thick silicon nitride $\left(\mathrm{Si}_{3} \mathrm{~N}_{4}\right)$ membranes. STXM measurements on such samples were performed with the STXM at beamline BL5.3.2 of the Advanced Light Source (ALS) with a lateral resolution of $\sim 40 \mathrm{~nm} .{ }^{9}$ Scattering measurements on identically prepared or the very same samples were performed at beamline BL6.3.2 of the ALS 

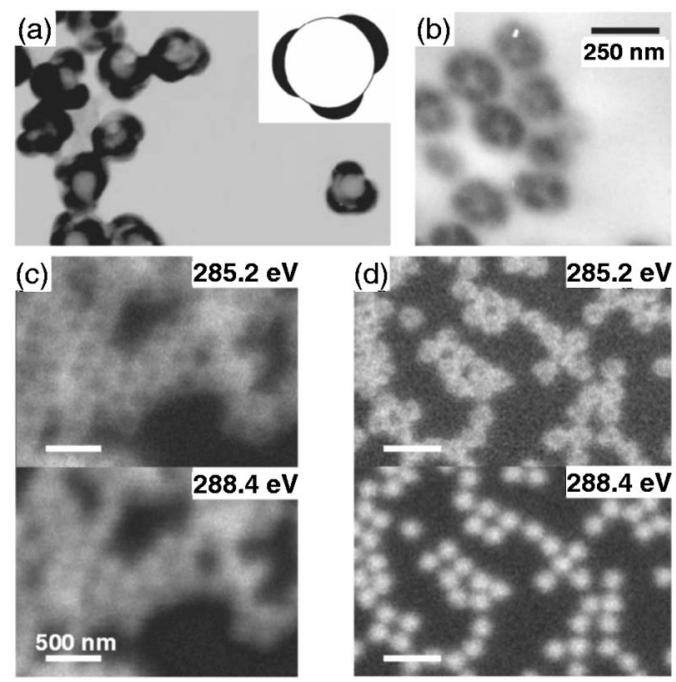

FIG. 1. (a) TEM image of sectioned PMMA/P(BA-co-S). (b) TEM image of sectioned P(MA-co-MMA)/PS. (c) STXM images in optical density scale of microtomed thin sections of PMMA/P(BA-co-S). (d) STXM images in optical density scale of whole particles of PMMA/P(BA-co-S).

(Ref. 22) in transmission. Near edge X-ray absorption fine structure (NEXAFS) spectra of PS and PMMA were acquired at BL5.3.2. These absorption spectra were converted to $\beta$, the absorptive part of the complex index of refraction $(n=1-\delta-i \beta)$, by scaling the pre- and postedges to the Henke database. ${ }^{23}$ The dispersion $\delta$ was calculated from $\beta$ through Kramers-Kronig integral relations. ${ }^{18}$ Both samples were sectioned for TEM and STXM analyses. For TEM, the sections were also stained in ruthenium oxide $\left(\mathrm{RuO}_{4}\right)$ vapor for selective PS contrast enhancement.

Sample A, PMMA/P(BA-co-S), was previously characterized extensively. ${ }^{6}$ The consensus "popcorn-type" shape has $\mathrm{P}(\mathrm{BA}-\mathrm{Co}-\mathrm{S})$ lobes protruding from a PMMA core and is shown schematically in Fig. 1(a), along with TEM results of one of the sample preparations described by Stubbs and Sundberg. ${ }^{6}$ Sample B is not as well characterized. TEM of $\mathrm{RuO}_{4}$ stained sections suggests that the PS [dark region in Fig. 1(b)] was distributed more towards the outside region of the particle. Yet the delineation of the outside edge and the separation between PS and P(MA-co-MMA) are indistinct. With NEXAFS microscopy, a core-shell-type structure can be observed in the thin section and for the whole particles of sample A [Figs. 1(c) and 1(d), respectively]. The mottled nature and the larger size of the $\mathrm{P}(\mathrm{S}-\mathrm{co}-\mathrm{BA})$ signature in Fig. $1(\mathrm{~d})$ corresponds to the morphology of $\mathrm{P}(\mathrm{S}-\mathrm{co}-\mathrm{BA})]$ lobes on a PMMA core, as indicated schematically in Fig. 1(a). In contrast, whole particles of sample B, P(MA-co-MMA)/PS, did not show any contrast in images acquired using identical STXM settings. Images at $532.2 \mathrm{eV}$, an energy corresponding to $\mathrm{O} 1 s \rightarrow \pi^{*}{ }_{\mathrm{C}=\mathrm{O}}$ transitions, also showed no contrast. STXM data for sample B are thus not shown here. Calorimetry of sample B shows that these particles consist, however, of polymer domains that are well phase separated. Hence, the STXM and TEM observations must reflect the distribution and small size scale of these domains and the limited chemical sensitivity, respectively.

The compositional sensitivity of RXS can be appreciated from Fig. 2. Figures 2(a) and 2(b) show the $\beta$ (absorption) and $\delta$ (dispersion) of PS and PMMA in the vicinity of the carbon $1 s$ absorption edge, respectively. Two sharp charac-

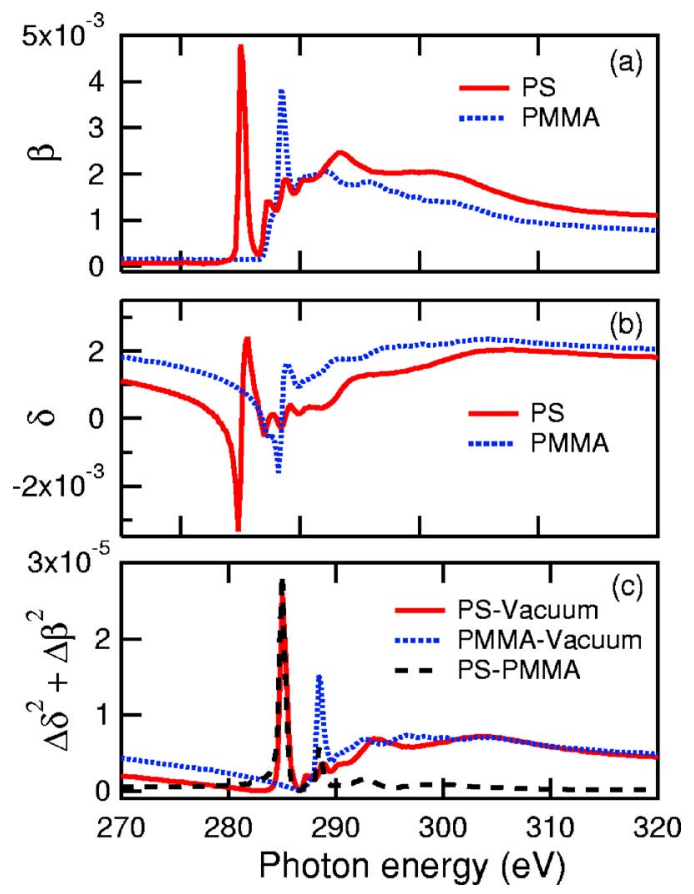

FIG. 2. (Color online) (a) Absorptive and (b) dispersive terms of the complex index of refraction near the carbon $K$-edge photon energy region. (c) Calculated scattered intensity for PS, PMMA, and vacuum. (d) Calculated scattering intensity for PS and PMMA. [Solid line represents PS and the dashed line represents PMMA in (a)-(c).]

teristic peaks at $285.2 \mathrm{eV}\left(\mathrm{C} 1 s \rightarrow \pi^{*}{ }_{\mathrm{C}=\mathrm{C}}\right.$ transitions in the phenyl ring of PS) and $288.4 \mathrm{eV}\left(\mathrm{C} 1 s \rightarrow \pi^{*}{ }_{\mathrm{C}=\mathrm{O}}\right.$ transitions in the carbonyl moiety in PMMA) can be observed for $\beta .^{8}$ Poly(butyl acrylate) and poly(methyl acrylate) have NEXAFS spectra similar to PMMA, but with slightly different $\mathrm{C}$ $1 s \rightarrow \pi^{*}{ }_{\mathrm{C}=\mathrm{O}}$ intensities. For the present discussion, we will use the PMMA NEXAFS spectrum as the prototypical acrylate NEXAFS spectrum. The X-ray scattered intensity is proportional to $|\delta(E)+i \beta(E)||\delta(E)-i \beta(E)|$ and is at high photon energies dominated by electron density differences. Near resonance, the intensity is modulated strongly as shown in Figs. 2(c) and 2(d), providing energy dependent, "moietyspecific" scattering. These RXS features should have a characteristic signature for a wide range of materials, similar to and related to the well documented sensitivity of carbon NEXAFS to composition in carbonaceous materials. ${ }^{24,25}$

Resonant scattering experiments of samples A and B were carried out at three photon energies that were selected to provide resonant enhancement for styrene $(285.2 \mathrm{eV})$ and acrylics $(288.4 \mathrm{eV})$, and for scattering of polymer particles "as a whole" (320.0 eV). Figures 3 show scattered intensity at these three photon energies versus $q$. Well defined oscillations can be observed for both samples due to the relatively monodisperse size distribution of these particles. Irrespective of the shape of particles, the low $q$ maxima and minima in these oscillations are related to the effective radius. Interestingly, the maxima and minima depend strongly on the photon energy, reflecting the effective compositional radial distribution of the nanoparticles. ${ }^{26}$ The scattering of sample A indicates that the effective radius corresponding to the PS phases is larger than the one corresponding to the PMMA phase. The corresponding minima and maxima for the $285.2 \mathrm{eV}$ data, dominated by the PS signal, are at significantly lower $q$ values than the minima and maxima for photon energies of AIP license or copyright; see http://apl.aip.org/apl/copyright.jsp 


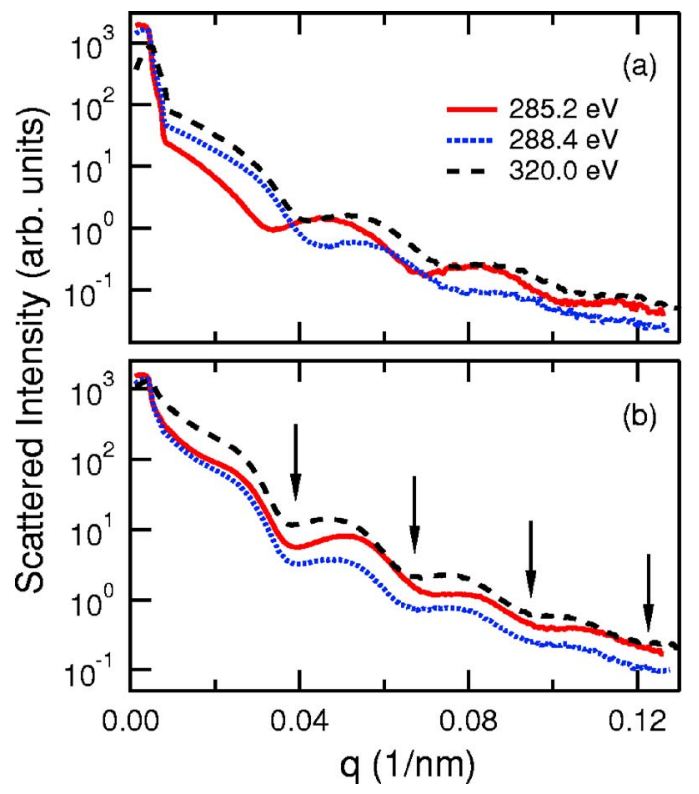

FIG. 3. (Color online) Scattered intensity of (a) PMMA/P(BA-co-S) and (b) $\mathrm{P}(\mathrm{MA}-\mathrm{co}$-MMA)/PS at $285.2 \mathrm{eV}$ (solid lines), $288.4 \mathrm{eV}$ (dotted lines), and $320 \mathrm{eV}$ (dashed lines). The theoretical scattering minima of dispersed ideal spheres of radius $R$ (occurring for $q R=4.5,7.73,10.9$, and 14.1) are indicated for $R=115 \mathrm{~nm}$. This radius matches well with the independently derived size for these particles (Ref. 6).

288.4 or $320 \mathrm{eV}$. This is consistent with prior knowledge of sample A with a well defined PMMA core and a styrene-rich "lobed shell." In contrast to this, the scattering minima and maxima for sample B do not exhibit a strong dependence on photon energy. Furthermore, the minima in the $288.4 \mathrm{eV}$ data are at smaller $q$ values than in the 285.2 or the $320 \mathrm{eV}$ data. The styrenic and acrylic phases have about the same effective radii with maybe a slight acrylic excess on the surface. Thus, sample B does not have a clearly defined average coreshell structure. A more detailed analysis would require use of more sophisticated models. The semiquantitative discussion presented is sufficient to demonstrate the complementary analysis capabilities of RXS.

The application of RXS will not be limited to the materials employed here. The extension of the technique to structured materials for which there are no TEM staining methods (e.g., structured acrylic based nanoparticles) and to multiphasic materials in general is possible. In addition, RXS scattering is a "photon-in and photon-out" technique, and control of the sample condition including in situ liquid environments and temperature control will be similar to that already achieved in STXM. ${ }^{27}$ The availability of several scattering profiles will also much better constrain sophisticated models of the samples than if only a single profile, as in neutron or hard $\mathrm{x}$-ray scattering, is available.

In summary, we demonstrated the compositional sensitivity of RXS and its utility for the characterization of nanoscale structured polymeric materials. Conventional XS would not have been able to assess effective radii, and neither STXM nor TEM was able to do so. Neutron scattering would have required the use of deuterated species. RXS thus has the potential to become a powerful complement to neutron and conventional x-ray scattering, particularly for polymeric and biological composite particles, vesicles, micelles, multiblock copolymers, various colloids and microgels, miscible polymer blends, polymer solutions, microcapsules, and lipid membranes, all of which are primarily composed of low $\mathrm{Z}$ elements with high, tunable contrast near their $K$-shell absorption edges.

The authors are grateful for the help of Tolek Tyliszczak, Eric Gullikson, Andrew Aquila, and Franklin Dollar. Data were acquired at BL5.3.2 and BL6.3.2 at the ALS, which is supported by the U.S. Department of Energy under Contract No. DE-AC02-05CH11231. Work at NCSU is supported by the U.S. Department of Energy (DE-FG02-98ER45737).

${ }^{1}$ D. C. Sundberg and Y. G. Durant, Polym. React. Eng. 11, 379 (2003).

${ }^{2}$ F. J. Balta-Calleja and C. G. Vonk, X-Ray Scattering of Synthetic Polymers (Elsevier, Amsterdam, 1989).

${ }^{3}$ B. Chu and B. S. Hsiao, Chem. Rev. (Washington, D.C.) 101, 1727 (2001).

${ }^{4}$ R. J. Roe, Methods of X-Ray and Neutron Scattering in Polymer Science (Oxford University Press, New York, 2000).

${ }^{5}$ C. K. Loong, P. Thiyagarajan, and A. I. Kolesnikov, Nanotechnology 15, S664 (2004).

${ }^{6}$ J. M. Stubbs and D. C. Sundberg, Polymer 46, 1125 (2005).

${ }^{7}$ H. Ade, X. Zhang, S. Cameron, C. Costello, J. Kirz, and S. Williams, Science 258, 972 (1992).

${ }^{8}$ H. Ade, Trends Polym. Sci. 5, 58 (1997).

${ }^{9}$ A. L. D. Kilcoyne, T. Tyliszczak, W. F. Steele, S. Fakra, P. Hitchcock, K. Franck, E. Anderson, B. Harteneck, E. G. Rightor, G. E. Mitchell, A. P. Hitchcock, L. Yang, T. Warwick, and H. Ade, J. Synchrotron Radiat. 10, 125 (2003)

${ }^{10} \lambda=1239.9 \mathrm{~nm} / E(\mathrm{eV})$. For $E=300 \mathrm{eV}, \lambda / 2=2.07 \mathrm{~nm}$; for $E=540 \mathrm{eV}$, $\lambda / 2=1.15 \mathrm{~nm}$.

${ }^{11}$ O. Glatter and O. Kratky, Small Angle X-Ray Scattering (Academic, New York, 1982).

${ }^{12}$ S. H. Anastasiadis, T. P. Russell, S. K. Satija, and C. F. Majkrzak, J. Chem. Phys. 92, 5677 (1990).

${ }^{13}$ M. L. Fernandez, J. S. Higgins, J. Penfold, R. C. Ward, C. Shackleton, and D. J. Walsh, Polymer 29, 1923 (1988).

${ }^{14}$ W. A. Hendrickson, Science 254, 51 (1991).

${ }^{15}$ D. Gibbs, D. R. Harshman, E. D. Isaacs, D. B. Mcwhan, D. Mills, and C. Vettier, Phys. Rev. Lett. 61, 1241 (1988).

${ }^{16}$ P. H. Fuoss, P. Eisenberger, W. K. Warburton, and A. Bienenstock, Phys. Rev. Lett. 46, 1537 (1981).

${ }^{17}$ H. A. Dürr, E. Dudzik, S. S. Dhesi, J. B. Goedkoop, G. van der Laan, M. Belakhovsky, C. Mocuta, A. Marty, and Y. Samson, Science 284, 2166 (1999).

${ }^{18}$ J. B. Kortright and S. K. Kim, Phys. Rev. B 62, 12216 (2000).

${ }^{19}$ C. Wang, T. Araki, and H. Ade, Appl. Phys. Lett. 87, 214109 (2005).

${ }^{20}$ G. E. Mitchell, B. G. Landes, J. Lyons, B. J. Kern, M. J. Devon, I. Koprinarov, E. M. Gullikson, and J. B. Kortright, Appl. Phys. Lett. 89, 044101 (2006).

${ }^{21}$ C. F. Welch, R. P. Hjelm, J. T. Mang, D. A. Wrobleski, E. B. Orler, M. E. Hawley, and J. B. Kortright, Polym. Mater. Sci. Eng. 93, 289 (2005).

${ }^{22}$ J. H. Underwood and E. M. Gullikson, J. Electron Spectrosc. Relat. Phenom. 92, 265 (1998).

${ }^{23}$ B. L. Henke, E. M. Gullikson, and J. C. Davis, R. L. Shimabukuro, and B. K. Fujikawa, At. Data Nucl. Data Tables 54, 181 (1993).

${ }^{24} \mathrm{O}$. Dhez, H. Ade, and S. Urquhart, J. Electron Spectrosc. Relat. Phenom. 128, 85 (2003).

${ }^{25}$ S. G. Urquhart and H. Ade, J. Phys. Chem. B 106, 8531 (2002).

${ }^{26}$ Although the radius of gyration of a particle is well defined, we have chosen here to use the term "effective radius" for the particle instead in order to avoid any confusion with the radius of gyration of an individual polymer chain.

${ }^{27}$ G. E. Mitchell, L. R. Wilson, M. T. Dineen, S. G. Urquhart, F. Hayes, E. G. Rightor, A. P. Hitchcock, and H. Ade, Macromolecules 35, 1336 (2002). 